\title{
Management of oropharyngeal candidiasis with localized oral miconazole therapy: efficacy, safety, and patient acceptability
}

This article was published in the following Dove Press journal:

Patient Preference and Adherence

I8 July 201 I

Number of times this article has been viewed

\author{
Curtis D Collins' \\ Sarah Cookinham ${ }^{2}$ \\ Jeannina Smith ${ }^{2}$ \\ 'Department of Pharmacy Services, \\ ${ }^{2}$ Department of Medicine, Division \\ of Infectious Disease, University of \\ Michigan Health System, Ann Arbor, \\ MI, USA
}

Correspondence: Curtis D Collins UHB2D30 I/0008, Department of Pharmacy Services University of Michigan Health System I500 E. Med Ctr Dr, Ann Arbor, MI 48109, USA

Tel +l 7349368210

Fax +I 7349367027

Email cdcollin@med.umich.edu

\begin{abstract}
Oropharyngeal candidiasis is a very common localized infection of the mucus membranes of the oropharynx that is most commonly caused by the patient's own commensal Candida albicans. It is the most common opportunistic infection affecting patients with the human immunodeficiency virus (HIV) and is also quite common in patients with hematological malignancies. Effective treatment options are of high importance given the worldwide incidence of these disease states and the potential for development of oropharyngeal candidiasis in these patients. Various systemic and topical treatment options for patients with oropharyngeal candidiasis have existed for many years. Miconazole buccal tablets have recently been approved by the US Food and Drug Administration for the treatment of oropharyngeal candidiasis Clinical trials have demonstrated noninferiority in the treatment of oropharyngeal candidiasis when compared with clotrimazole troches in patients with HIV and against miconazole gel in patients with head and neck cancer. Miconazole buccal tablets exhibit few drug interactions because of low systemic absorption and are generally well tolerated with a safety profile similar to comparators. The once-daily dosing schedule may improve patient adherence compared with topical alternatives; however, the cost of therapy may be a barrier for some patients and should be considered by prescribers compared with alternative treatments.
\end{abstract}

Keywords: esophageal candidiasis, miconazole, antifungal agents

\section{Introduction}

Oropharyngeal candidiasis (OPC) is a very common localized infection of the mucus membranes of the oropharynx caused by Candida species. Most commonly, it is caused by the patient's own commensal Candida albicans, but it may also be caused by other Candida species such as C. glabrata, C. tropicalis, and C. krusei. ${ }^{1}$ There are several clinical variants of OPC. Type one OPC, or pseudomembranous OPC, is also known as "thrush". Thrush is characterized by white curd-like discrete plaques on an erythematous base found on the buccal mucosa, throat, tongue, or gingivae. These plaques rub off and reveal a tender red surface underneath. Type two OPC is the erythematous variant. In erythematous OPC there are smooth red patches on the hard or soft palate, dorsum of tongue, or buccal mucosa. Denture-induced stomatitis may also be considered a variant of erythematous OPC and is seen in $65 \%$ of denture wearers, presenting as either a smooth or granular erythema confined to the denture-bearing area of the hard palate. ${ }^{2}$ The third OPC type is the hyperplastic variant. This consists of white, firmly adherent patches on the bilateral buccal mucosa, tongue, or palate. Finally, angular cheilitis, which is a red fissured lesion at the corners of the mouth, is also considered part of OPC. ${ }^{3}$ Infection can be minimally to very symptomatic. 
Symptoms include mouth irritation and a burning sensation on the tongue. It may also cause perturbations of taste and ability to eat and/or speak.

OPC is the most common opportunistic fungal infection among patients infected with human immunodeficiency virus (HIV). ${ }^{4}$ OPC also affects patients with cancer. A systematic review of oral fungal infections in patients receiving cancer treatment found a weighted prevalence of $39.1 \%$ for patients undergoing cancer treatment. ${ }^{5}$ However, unlike esophageal candidiasis, which is only seen in the setting of major derangements of cell-mediated immunity such as AIDS, immunosuppression for transplantation or chemotherapy, OPC is also associated with very small derangements in the host's local immune response, such as that seen with the use of topical or systemic antibacterial therapy, inhaled or systemic steroids, and diabetes.

A variety of options exist for the treatment of OPC. This article focuses on one of the newest additions to our antifungal armamentarium, miconazole buccal tablets

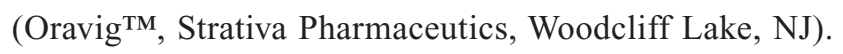
The FDA licensure of Oravig (miconazole) for the treatment of oropharyngeal candidiasis occurred in April 2010, with the majority of clinical evidence considered for FDA approval coming from observations and trials in humans conducted between 2000 and 2004. ${ }^{6}$ Miconazole buccal tablets are currently the only oral preparation of miconazole licensed in the USA for treatment of oropharyngeal candidiasis. Outside the USA, miconazole oral gel (MOG), marketed under the trade name Daktarin ${ }^{\mathrm{TM}}$ (Janssen Pharmaceutica, Beerse, Belgium), has been available for the treatment of oral and gastrointestinal fungal infections since 1977. The European Union also recently approved oral miconazole buccal tablets (Loramyc ${ }^{\mathrm{TM}}$, BioAlliance Pharma, Paris, France) for the treatment of oropharyngeal candidiasis in immune-compromised patients in 2007. Miconazole buccal tablet safety data exists only for persons over the age of 16 years, while use of MOG extends to those greater than 4 months. MOG and other antifungal preparations are mentioned here because trials comparing these have factored into the decision for licensing and use in the USA.

\section{Mechanism of action}

Miconazole is a broad-spectrum triazole antifungal available in the USA since the 1970s.

Miconazole works at the cell membrane level by limiting ergosterol synthesis through inhibiting the cytochrome P450 14 $\alpha$-demethylase enzyme. Miconazole also affects the synthesis of triglycerides and fatty acids and inhibits oxidative and peroxidative enzymes. ${ }^{7}$

Miconazole, like other triazole antifungals, has long exhibited in vitro activity against Candida species, including C. albicans, C. glabrata, and C. krusei; however, miconazole resistance has been reported in up to $17 \%$ of $C$. albicans and $45 \%$ in non-albicans species. ${ }^{8}$ The mechanism of resistance is thought to be due to mutations in the EFG11 gene, expressions of transporter proteins functioning as efflux pumps coded by CDR1 and CDR2 genes, or to major facilitator genes. ${ }^{9,10}$

A recent in vitro analysis studied miconazole susceptibilities utilizing Clinical Laboratory and Susceptibility standards with current clinical isolates. Miconazole was shown to retain activity against most Candida species. The authors also reported that miconazole had a 12 -fold lower minimum inhibitory concentration to inhibit the growth of $90 \%$ of organisms (minimum inhibitory concentration [MIC] 90) than fluconazole and exhibited activity against fluconazole resistant strains. ${ }^{11}$ Six Candida isolates, including two fluconazole resistant strains, were subsequently tested by the same authors for the development of resistance to miconazole. Two isolates experience an increase in MIC, though no isolate acquired resistance to miconazole. ${ }^{12}$ Despite this positive evidence, antimicrobial resistance concerns, including miconazole, remain an important consideration when selecting treatments for OPC.

A pharmacokinetic study of 18 healthy volunteers showed a mean maximum salivary concentration of $15 \mathrm{mcg} / \mathrm{mL}$ at 7 hours following placement of the tablet. An average saliva exposure to miconazole of $55.23 \mathrm{mcg}$. hours $/ \mathrm{mL}$ was estimated from the AUC ( $0-24$ hours). The terminal half-life of miconazole buccal tablets is 24 hours. ${ }^{13}$

The systemic absorption of miconazole buccal tablets is limited. Plasma concentrations were below the lower limit of quantification $(0.4 \mathrm{mcg} / \mathrm{mL})$ in $157 / 162$ (97\%) samples from healthy volunteers following single-dose application. Measurable plasma concentrations ranged from 0.5 to $0.83 \mathrm{mcg} / \mathrm{mL}$. Following 7 days of therapy in $40 \mathrm{HIV}$-positive patients, plasma concentrations of miconazole were below the detectable limit $(0.1 \mathrm{mcg} / \mathrm{mL})$. The manufacturer recommends caution in patients with hepatic dysfunction, despite limited systemic absorption. Miconazole buccal tablets are excreted unchanged less than $1 \%$ in the kidneys. As such, renal dosing adjustments are not needed. ${ }^{7}$

Miconazole is an inhibitor of CYP3A4 and CYP2C9. Despite limited systemic absorption with the buccal tablet formulation, the potential exists for drug-drug interactions 
with agents such as phenytoin, oral hypoglycemics and ergot alkaloids. Cases of bleeding and bruising have been reported following administration of oral miconazole in patients also taking warfarin. It is recommended to monitor closely for evidence of bleeding, prothrombin time, and international normalized ratio (INR) if a patient is taking both medications concominantly. ${ }^{7}$

Miconazole buccal tablets are contraindicated in patients with known hypersensitivity (eg, anaphylaxis) to miconazole, milk protein concentrate, or any other component of the product. They are listed as a pregnancy category $\mathrm{C}$ medication and should be avoided unless the risk to the mother outweighs risk to the fetus. It is not known whether oral miconazole is excreted in breast milk and there have been no studies to date in patients under the age of $16 .^{7}$

\section{Dosing and patient instructions}

Patients are instructed to apply the rounded side of the $50 \mathrm{mg}$ tablet to the upper gum region just above the right or left incisor following brushing of teeth in the morning. The tablet should be held in place until dissolved and should not be chewed, swallowed, or crushed. There are no restrictions on normal eating and drinking; however, the chewing of gum and wearing of upper dentures should be avoided. The average duration of adhesion was 15 hours in healthy volunteer trials. If the tablet falls off or does not adhere within 6 hours it is recommended the patient replace the tablet. It the tablet is swallowed within 6 hours, patients should drink a glass of water and apply a new tablet only once. If the tablet falls off or is swallowed after 6 hours the patient should wait until the next scheduled dose to apply a new tablet.?

\section{Early clinical studies of safety and tolerability}

Chaumont et al first examined safety and tolerability of novel slow-release miconazole buccal tablets in healthy individuals in 2001. ${ }^{14}$ Published before the manuscript of Cardot et al ${ }^{13}$ containing specific pharmacokinetics data from the same subjects and study period, the Chaumont Phase I study compared the $100 \mathrm{mg}$ and the $50 \mathrm{mg}$ miconazole buccal tablets, and miconazole oral gel (Daktarin). Eighteen subjects, ages 18-35, received each of the three treatments (one $50 \mathrm{mg}$, one $100 \mathrm{mg}$, or three applications of gel over 8.5 hours) in random order, with each treatment followed by a 1-week washout period. ${ }^{13,14}$ No adverse events were recorded with the $50 \mathrm{mg}$ miconazole buccal tablet; bad taste was described by one participant in relation to the $50 \mathrm{mg}$ tablet (as opposed to 13 reports with gel use). Discomfort was reported with the $100 \mathrm{mg}$ tablet, presumably secondary to tablet size and not localized chemical irritation.

\section{Clinical studies and efficacy}

Using a distinct miconazole buccal tablet size, Van Roey et al published a study in 2004 of 357 HIV-infected adults in Uganda who were randomized to receive either a once-daily $10 \mathrm{mg}$ slow-release miconazole buccal tablet or a $400 \mathrm{mg}$ daily oral dose of ketoconazole for treatment of clinically diagnosed oropharyngeal candidiasis. ${ }^{15}$ Treatment was provided for 7 or 14 days, depending on response at day 7 . The primary outcome studied was clinical cure, defined as a complete absence of symptoms and signs. Using per protocol analysis, the $10 \mathrm{mg}$ tablet demonstrated noninferiority to systemic ketoconazole for oropharyngeal candidiasis, with reported cure rates at end of treatment of $93 \%$ (155/167) and 96\% (159/165), respectively. Microscopic examination for fungal elements was negative in a slightly greater proportion of ketoconazole than miconazole buccal tablet recipients (75\% compared with $70 \%$; baseline negative smear rate of $23 \%$ in both groups) at end of treatment.

Two subsequent prospective randomized clinical trials involving the currently available $50 \mathrm{mg}$ tablet size form the basis of efficacy and noninferiority data (as compared to alternative oral antifungal preparations) to date for use of miconazole buccal tablets. Bensadoun et al in 2002 evaluated 282 adult patients receiving radiotherapy for head and neck cancer and oropharyngeal candidiasis (defined both by consistent clinical exam and positive fungal culture), who were randomly triaged to receive either once-daily $50 \mathrm{mg}$ MBT or $125 \mathrm{mg}$ miconazole gel applied four times daily for 14 days. ${ }^{16}$ Noninferiority $(P<0.0001)$ was established for the miconazole buccal tablet in a modified intent to treat analysis. The primary endpoint studied was treatment success, which was defined by a complete or partial clinical response (independent of mycologic data). Comparatively, $56 \%$ of the miconazole treated subjects and $48.9 \%$ of the gel users had achieved clinical success; symptomatic improvement was also not statistically significant between the two groups $(70.3 \%$ and $76.5 \%$ respectively).

The SMiLES (study of miconazole Lauriad ${ }^{\circledR}$ efficacy and safety) published in 2010, examined 578 adult HIV-infected patients (at multiple international sites) with clinical evidence of oropharyngeal candidiasis accompanied by positive $\mathrm{KOH}$ smears and fungal cultures, who were randomized to receive 14 days of once-daily active $50 \mathrm{mg}$ miconazole buccal tablets plus placebo troches five times daily or once-daily placebo 
buccal tablets plus active $10 \mathrm{mg}$ clotrimazole troches five times daily. ${ }^{17}$ The primary endpoint was clinical cure defined as complete resolution of signs and symptoms; additional data on the secondary endpoints of clinical success (clinical cure or improvement) and mycologic cure were included. Using an intent-to-treat analysis, $61 \%$ of miconazole-treated patients compared with $65 \%$ of clotrimazole-troche treated patients attained the primary endpoint of clinical cure. Mycologic cure (27\% with miconazole tablets, 25\% with clotrimazole), clinical success, and rates of relapse were not statistically significant between the two groups.

\section{Adherence}

In those treated with miconazole buccal tablets for oropharyngeal candidiasis in the clinical context of radiotherapy for head and neck cancer, Bensadoun et al found that $6 \%$ of subjects were considered noncompliant ( $>3$ missed doses); adherence in the comparison miconazole gel-treated subjects was incompletely assessed and not specifically reported. ${ }^{16}$ Within the SMiLES trial, overall compliance with therapy was $87.2 \%$ for the miconazole buccal tablet group and $87.1 \%$ in the clotrimazole group. ${ }^{17}$ Adherence was determined by the number of unused tablets or troches returned at day 14 (end of treatment) and through daily self-administered questionnaires. A subset of 40 participants (20 from the miconazole tablet group and 20 from the clotrimazole group) underwent testing of miconazole levels at day 7 , but systemic levels were undetectable (below the sensitivity of the assay) in all 40 subjects.

\section{Adverse events}

Bensadoun et al reported the number of adverse events was similar among users of miconazole buccal tablets and miconazole gel, leading to discontinuation of drug in 3/141 and 6/141 study participants, respectively. Tolerability was assessed through a self-administered questionnaire, notably with $6.1 \%$ of miconazole tablet-treated patients reporting dysgeusia as opposed to $0 \%$ of gel-treated patients. Adverse events (diarrhea, headache, nausea and vomiting being the most frequent) in the SMiLES study were comparable between its study groups, with no statistically significant difference in overall rates for miconazole buccal tablet- or clotrimazole-treated participants. When collectively considered by expert reviewers, none of the serious adverse events (including death, anemia, lower respiratory tract infection) reported in trials have been directly attributed to oral miconazole buccal tablets. ${ }^{14}$ With any of the available local antifungal treatments for oropharyngeal candidiasis, listed side effects include nausea, diarrhea, headache and dysgeusia. Specific to the miconazole buccal tablets, local site reactions, such as oral discomfort (5\%), burning $(7.2 \%)$, bad taste $(7.2 \%)$ and pain (1.8\%), are reported but very infrequently lead to discontinuation of drug use. ${ }^{16,17}$

Potential drug interactions with miconazole (and the relevance of miconazole to inhibit CYP2C9 and CYP3A4) are discussed elsewhere, but it should be noted that liver function test (LFT) abnormalities were infrequently encountered ( $<2 \%$ of cumulative study patients experiencing a three-fold increase in transaminases or alkaline phosphatase above baseline) and comparable to other localized antifungal therapies for oropharyngeal candidiasis (clotrimazole troches, nystatin suspension). ${ }^{14}$ A potential choking hazard exists for a drug with this formulation and mode of administration (obviating the appropriateness of use in young children), but specific events of this type have not been reported in the current literature.

\section{Place in therapy}

OPC can be effectively treated with topical or systemic antifungal therapy. There are distinct advantages and disadvantages to both types of treatment. The preferred treatment of OPC often differs by patient population, with systemic agents more often used in immunosuppressed patients than the topical options. There are several other factors that may also affect treatment choice including infection type and severity, presence of Candidal infection elsewhere (vulvovaginal, intertrigo), drug efficacy, adverse effects, ease and frequency of administration, anticipated adherence, gastric acidity (which may affect absorption), possible systemic toxicity, drug-drug interactions, and cost.

Oral fluconazole is first-line systemic therapy for OPC. Treatment with oral fluconazole 200 or $100 \mathrm{mg}$ daily is easy and highly efficacious. A recent trial also suggests that a single dose of oral fluconazole $750 \mathrm{mg}$ has equal efficacy and relapse rates. ${ }^{18}$ Itraconazole, voriconazole, and posaconazole also have efficacy in the treatment of OPC. However, these agents would typically only be used as salvage therapy in the setting of resistance or OPC with a non-albicans Candida species. ${ }^{19,20}$ The Infectious Disease Society of America (IDSA) guidelines suggest itraconazole solution if fluconazole failure occurs. This strategy is effective $64 \%-80 \%$ of the time. ${ }^{21}$ Echinocandins may also be useful in this setting especially because they have a different mechanism of action than the azoles and may be used in cases of high-level azole resistance. ${ }^{22}$ 
As noted previously, systemic therapy is most often used in patients who are significantly immune compromised. Systemic therapy is also useful when there is concern that the infection extends past the oropharynx into the esophagus or elsewhere in the body, such as the skin folds or genitals. The major disadvantage of systemic therapy is the greater potential for drug-drug interactions, and systemic toxicity such as hepatotoxicity with systemic use. Though some degree of drug-drug interaction is noted with oral miconazole, systemic drug levels are typically undetectable. ${ }^{7}$ Though it has not been studied in a randomized manner, it is therefore likely that the incidence of systemic adverse effects would be less, as many of the adverse effects of azoles are level related. ${ }^{23}$

When comparing miconazole buccal tablets to the other topical agents, miconazole has the advantage of being applied once daily versus five times a day with clotrimazole troche and four times daily with nystatin swish and swallow. ${ }^{24}$ Adherence has been shown to be greatly enhanced with less frequent dosing of all pharmacological agents and this may therefore represent a potential advantage. The limited available adherence data, discussed previously, supports at least comparable rates for miconazole buccal tablets and alternative local therapies for oropharyngeal candidiasis but not clear superiority favoring miconazole buccal tablets. At least one international organization has weighed in on the role of miconazole buccal tablets for the treatment of OPC. The World Health Organization (WHO) recently reviewed miconazole buccal tablets for the treatment of OPC for inclusion on their WHO Model Lists of Essential Medicines. They determined that evidence for clinical benefits arising from a potential improved patient adherence was not available and rejected the proposal on the basis of inadequate comparison data with nystatin formulations. ${ }^{25}$

Because of the need for a suitable location to apply the adhesive tablet, miconazole buccal tablets may not be as useful as the other agents in denture-induced stomatitis. Angular cheilitis is best treated with topical antifungal creams or ointments. ${ }^{26}$

\section{Cost considerations}

Cost is an important consideration for the treatment of OPC due to the availability of generic or relatively low-cost treatment options. The cost of oral miconazole buccal tablets for a 14-day treatment course is considerable (Table 1). As with many of the other OPC treatment options, we are unaware of any published pharmacoeconomic studies performed to date evaluating miconazole buccal tablets with less-expensive
Table I Medication costs for the treatment of oropharyngeal candidiasis

\begin{tabular}{lll}
\hline Medication & Dosage & $\begin{array}{l}\text { I4-day cost } \\
\text { (US } \$ \text { ) }\end{array}$ \\
\hline Miconazole buccal tablets & & $\$ 270$ \\
Clotrimazole troches $^{28}$ & $50 \mathrm{mg} /$ day & $\$ 204$ \\
Nystatin oral suspension & $10 \mathrm{mg}, 5 \times /$ day & $\$ 69$ \\
Fluconazole oral tablets & $200 \mathrm{~mL}, 4 \times /$ day & I, then \\
& $100 \mathrm{mg} /$ day & $\$ 125$ \\
\hline
\end{tabular}

alternatives. In general, further cost-effectiveness studies for the treatment of OPC are needed due to the worldwide incidence of HIV and the potential financial impact on the global community.

\section{Conclusion}

Oropharyngeal candidiasis is a localized infection of the mucous membranes and is an especially common opportunistic infection in patients with HIV. A variety of options, both systemic and topical, exist for the treatment of OPC. Miconazole buccal tablets are the most recent addition to the antifungal armamentarium available for the treatment of OPC. The convenient once-daily dosing may enhance patient adherence compared with existing topical options; however, the cost compared with both topical and systemic agents should be considered.

\section{Disclosure}

The authors have received no financial support for this review and declare no conflicts of interest in relation to this work.

\section{References}

1. Pankhurst CL. Candidiasis (oropharyngeal). Clin Evid (Online). 2009;2009:1304. Epub 2009 Mar 18.

2. Webb BC, Thomas CJ, Willcox MD, Harty DW, Knox KW. Candida-associated denture stomatitis. Aetiology and management: a review. Part 3. Treatment of oral candidosis. Aust Dent J. 1998;43(4):244-249.

3. Ellepola ANB, Samaranayake LP. Antimycotic agents in oral candidosis: an overview: 1. Clinical variants. Dent Update. 2000;27(3): 111-112,114-116.

4. Samaranayake LP. Oral mycoses in HIV infection. Oral Surg Oral Med Oral Pathol. 1992;73(2):171-180.

5. Lalla RV, Latortue MC, Hong $\mathrm{CH}$, et al. A systematic review of oral fungal infections in patients receiving cancer therapy. Support Care Cancer. 2010;18(8):985-992.

6. Center for Drug Evaluation and Research, US Food and Drug Administration (FDA). Summary Review: application 22404Orig1s000; NDA22404, Oravig (miconazole) for oropharyngeal candidiasis. US Center for Drug Evaluation and Research; 2010. Available at: http://www. accessdata.fda.gov/drugsatfda_docs/nda/2010/022404Orig1s000SumR. pdf. Accessed May 12, 2011.

7. Oravig [prescribing information]. Woodcliff Lake, NJ: Strativa Pharmaceuticals, a division of Par Pharmaceutical, Inc; 2010. Available at: www.oravig.com/oral-thrush-once-daily/hcp-faqs.html. Accessed May 12, 2011. 
8. Manfredi M, McCullough MJ, Polonelli L, et al. In vitro antifungal susceptibility to six antifungal agents of 229 Candida isolates from patients with diabetes mellitus. Oral Microbiol Immunol. 2006;21(3):177-182.

9. Luz Paniagua GC, Monroy EP, Negrete EA, Vaca SP. Susceptibility to 5-fluorocytosine, miconazole and amphotericin B of Candida albicans strains isolated from the throat of non-AIDS patients. Rev Latinoam Microbiol. 2002;44(2):65-68.

10. Hernáez ML, Gil C, Pla J, Nombela C. Induced expression of Candida albicans multidrug resistance gene CDR1 in response to fluconazole and other antifungals. Yeast. 1998;14(6):517-526.

11. Isham N, Ghannoum MA. Antifungal activity of miconazole against recent Candida strains. Mycoses. 2010;53(5):434-437.

12. Ghannoum MA, Herbert J, Isham N. Repeated exposure of Candida spp. to miconazole demonstrates no development of resistance. Mycoses. 2010 Mar 4. [Epub ahead of print]

13. Cardot JM, Chaumont C, Dubray C, Constantini D, Aiache JM. Comparison of the pharmacokinetics of miconazole after administration via a bioadhesive slow release tablet and an oral gel to healthy male and female subjects. Br J Clin Pharmacol. 2004;58(4): 345-351.

14. Chaumont C, Cardot JM, Dubray C, Constantini D, Aiache JM. Safety, acceptability, and adhesion of novel bioadhesive slow release tablet of miconazole in a phase I study. Journal de mycologie médicale. 2003; 13:13-18.

15. Van Roey J, Haxaire M, Kamya M, Lwanga I, Katabira I. Comparative efficacy of topical therapy with a slow-release mucoadhesive buccal tablet containing miconazole nitrate versus systemic therapy with ketoconazole in HIV-positive patients with oropharyngeal candidiasis. J Acquir Immune Defic Syndr. 2004;35(2): 144-150.

16. Bensadoun RJ, Daoud J, El Gueddari B, et al. Comparison of the efficacy and safety of miconazole $50 \mathrm{mg}$ mucoadhesive buccal tablets with miconazole $500 \mathrm{mg}$ gel in the treatment of oropharyngeal candidiasis: a prospective, randomized, single-blind, multicenter, comparative, phase III trial in patients treated with radiotherapy for head and neck cancer. Cancer. 2008;112(1):204-211.
17. Vazquez JA, Patton LL, Joel B, et al. Randomized, comparative, doubleblind, double-dummy, multicenter trial of miconazole buccal tablet and clotrimazole troches for the treatment of oropharyngeal candidiasis: study of miconazole Lauriad ${ }^{\circledR}$ efficacy and safety (SMiLES). HIV clinical trials. 2010;11(4):186-196.

18. Hamza OJ, Matee MI, Bruggemann RJ, et al. Single-dose fluconazole versus standard 2-week therapy for oropharyngeal candidiasis in HIVinfected patients: a randomized, double-blind, double-dummy trial. Clin Infect Dis. 2008;47(10):1270-1276.

19. Vazquez JA, Skiest DJ, Nieto L, et al. A multicenter randomized trial evaluating posaconazole versus fluconazole for the treatment of oropharyngeal candidiasis in subjects with HIV/AIDS. Clin Infect Dis. 2006;42(8):1179-1186.

20. Skiest DJ, Vazquez JA, Anstead GM, et al. Posaconazole for the treatment of azole-refractory oropharyngeal and esophageal candidiasis in subjects with HIV infection. Clin Infect Dis. 2007;44(4): 607-614.

21. Pappas PG, Kauffman CA, Andes D, et al. Clinical practice guidelines for the management of candidiasis: 2009 update by the Infectious Diseases Society of America. Clin Infect Dis. 2009;48(5): 503-535.

22. Villanueva, Arathoon EG, Gotuzzo E, Berman RS, DiNubile MJ, Sable CA. A randomized double-blind study of caspofungin versus amphotericin for the treatment of candidal esophagitis. Clin Infect Dis. 2001; 33(9):1529-1535.

23. Smith JA. Is there a role for therapeutic drug monitoring of antifungal agents? Curr Infect Dis Rep. 2009;11(6):439-446.

24. Klotz SA. Oropharyngeal candidiasis: a new treatment option. Clin Infect Dis. 2006;42(8):1187-1188.

25. Who Technical Report Series, No. 920. The selection and use of essential medicines. Available at: http://apps.who.int/medicinedocs/en/d/Js 4881e/4.1.5.html\#Js4881e.4.1.5. Accessed April 11, 2011.

26. Sharon V, Fazel N. Oral candidiasis and angular cheilitis. Dermatol Ther. 2010;23(3):230-242.

27. Miconazole (Oravig) for oropharyngeal candidiasis. Med Lett Drugs Ther. 2010;29;52(1352):95-96.

28. 2010 Redbook. Montvale, NJ: Thomson Healthcare; 2010.
Patient Preference and Adherence

\section{Publish your work in this journal}

Patient Preference and Adherence is an international, peer-reviewed, open access journal focusing on the growing importance of patient preference and adherence throughout the therapeutic continuum. Patient satisfaction, acceptability, quality of life, compliance, persistence and their role in developing new therapeutic modalities and compounds to

\section{Dovepress}

optimize clinical outcomes for existing disease states are major areas of interest. This journal has been accepted for indexing on PubMed Central. The manuscript management system is completely online and includes a very quick and fair peer-review system. Visit http://www.dovepress.com/ testimonials.php to read real quotes from published authors. 